\title{
Erythrocyte membrane trans-fatty acid index is positively associated with a 10-year CHD risk probability
}

\author{
Xiao-Ru Liu ${ }^{1,2}$, Ze-Yuan Deng ${ }^{1,2 *}$, Jiang-Ning Hu${ }^{1}$, Ya-Wei Fan ${ }^{1}$, Rong Liu ${ }^{1}$, Jing Li $^{1}$, Jing-Tian Peng ${ }^{3}$, \\ Hai $\mathrm{Su}^{4}$, Qiang Peng ${ }^{4}$ and Wei-Feng $\mathrm{Li}^{3}$ \\ ${ }^{1}$ State Key Laboratory of Food Science and Technology, Nanchang University, 235, East Nanjing Road, Nanchang, Jiangxi \\ Province, People's Republic of China \\ ${ }^{2}$ Institute for Advanced Study, Nanchang University, Nanchang, Jiangxi, People's Republic of China \\ ${ }^{3}$ Department of Cardiology, The First Affiliated Hospital, Nanchang University, Nanchang, Jiangxi, People's Republic of China \\ ${ }^{4}$ Department of Cardiology, The Second Affiliated Hospital, Medical College Nanchang University, Nanchang, Jiangxi, \\ People's Republic of China
}

(Submitted 15 May 2012 - Final revision received 3 January 2013 - Accepted 4 January 2013 - First published online 25 February 2013)

\section{Abstract}

Industry-generated trans-fatty acids (TFA) are detrimental to risk of CHD, but ruminant-originated TFA have been reported as neutral or equivocal. Therefore, the total TFA amount should not be the only factor considered when measuring the effects of TFA. In the present study, we addressed whether a version of the TFA index that unifies the effects of different TFA isomers into one equation could be used to reflect CHD risk probability (RP). The present cross-sectional study involved 2713 individuals divided into four groups that represented different pathological severities and potential risks of CHD: acute coronary syndrome (ACS, $n$ 581); chronic coronary artery disease (CCAD, $n$ 631); high-risk population (HRP, $n$ 659); healthy volunteers (HV, $n$ 842). A 10-year CHD RP was calculated. Meanwhile, the equation of the TFA index was derived using five TFA isomers (trans-16:1n-7, trans-16:1n-9, trans-18:1n-7, trans-18:1n-9 and trans-18:2n-6n-9), which were detected in the whole blood, serum and erythrocyte membranes of each subject. The TFA index and the 10-year CHD RP were compared by linear models. It was shown that only in the erythrocyte membrane, the TFA isomers were significantly different between the groups. In the ACS group, industry-generated TFA (trans-16:1n-9, trans-18:1n-9 and trans-18:2n-6n-9) were the highest, whereas ruminant-originated TFA (trans-16:1n-7 and trans-18:1n-7), which manifested an inverse relationship with CHD, were the lowest, and vice versa in the HV group. The TFA index decreased progressively from $7 \cdot 12$ to $5.06,3.11$ and 1.92 in the ACS, CCAD, HRP and HV groups, respectively. The erythrocyte membrane TFA index was positively associated with the 10-year CHD RP ( $R^{2}$ 0.9981) and manifested a strong linear correlation, which might reflect the true pathological severity of CHD.

\section{Key words: Trans-fatty: acid index: CHD: Erythrocyte membranes}

The effect of blood trans-fatty acid (TFA) levels on human diseases has recently aroused considerable attention ${ }^{(1-3)}$. Chavarro et $a l .{ }^{(4)}$ reported that the whole-blood TFA level was associated with an increased risk of non-aggressive prostate tumour. Chajes et al. ${ }^{(5)}$ showed that a high serum level of TFA contributed to the risk of invasive breast cancer in women. Benatar et $a l^{(6)}$ proposed that plasma total TFA may be associated with vascular disease and increased C-reactive protein in patients with severe coronary artery disease. Meanwhile, Lemaitre et al. ${ }^{(7)}$ found that a high erythrocyte membrane TFA level was correlated with an increased risk of sudden cardiac arrest.

It is well accepted that TFA are highly associated with CHD risk, and different kinds of TFA isomers play different roles in CHD events ${ }^{(8,9)}$. Industry-originated TFA, such as trans16:1n-9, trans-18:1n-9 and trans-18:2n-6n-9, are considered to have deleterious effects on cardiovascular health ${ }^{(2,10,11)}$, while TFA from ruminant sources are associated with a slightly neutral risk ${ }^{(12)}$, because trans-16:1n-7 and trans-18: $1 n-7$, the dominant TFA isomers in milk, can be biotransformed to conjugated linoleic acid (CLA) such as 9-cis, 11-trans-18:2n-6 CLA by $\Delta 9$-desaturase, which is conducive to anticancer, anti-diabetic and anti-CHD effects ${ }^{(13)}$. Therefore, the effects of TFA on CHD vary in differently originated TFA, and it may be irrational to estimate the effects of TFA simply by considering the total amount of TFA levels. However, if there were combined parameters, taking into account both industry-originated TFA and ruminant-sourced TFA, the effects

Abbreviations: ACS, acute coronary syndrome; CCAD, chronic coronary artery disease; CLA, conjugated linoleic acid; HRP, high-risk population; HV, healthy volunteers; RP, risk probability; TFA, trans-fatty acids.

*Corresponding author: Professor Z.-Y. Deng, fax +86 79188304402 , email zeyuandeng@hotmail.com 
of TFA on CHD could be estimated according to different TFA isomers and their own physico-chemical characteristics.

In the present study, we explored a TFA index that unifies the disparate impacts of different TFA isomers on CHD by discriminating the different effects into one equation. Meanwhile, a 10-year CHD risk probability (RP) was also calculated in the present cross-sectional study to represent different pathological severities and potential risks of CHD. The index may be used to confirm the presence of a true association between TFA and CHD. The aims of the present study were to verify whether the TFA index was associated reliably with the 10-year CHD RP and to identify which study sample (whole blood, serum or erythrocyte membrane) could truly reflect the change in body TFA levels.

\section{Methods}

\section{Ethical statement}

The present study was conducted according to the guidelines laid down in the Declaration of Helsinki, and all procedures involving human subjects/patients were approved by the Institutional Ethics Committee, the First and Second Affiliated Hospital, Nanchang University, China. Written informed consent was obtained from all subjects/patients.

\section{Patients}

In the present study, we divided CHD into two relatively independent clinical pathological forms: a severely harmful acute coronary syndrome (ACS), which is manifested by myocardial infarction and unstable angina, and a chronic/ stable form known as chronic coronary artery disease (CCAD), which has a phenotype of stable angina and ischaemic cardiomyopathy ${ }^{(14)}$. The ACS and CCAD patients were selected to represent an acute and a chronic CHD pathology, respectively. A high-risk population (HRP) and healthy volunteers (HV) served as controls for CHD patients in the ACS and CCAD groups. Thus, the four groups represented different pathological severities and potential risks of CHD.

A cross-sectional survey on six large physical populations of 12380 individuals was conducted at the First and Second Affiliated Hospitals, Nanchang University between 2007 and 2010. Excluding confounding factors and other diseases, 2713 individuals were screened in the ACS ( $n$ 581), CCAD ( $n$ 631), HRP ( $n$ 659) and HV ( $n$ 842) groups to represent different pathological severities and potential risks of CHD. The ACS was diagnosed on the basis of pre-specified criteria for acute myocardial infarction or unstable angina ${ }^{(15,16)}$. The CCAD patients were selected on the basis of the objective clinical and diagnostic criteria of the American Heart Association $^{(14,17)}$. The HRP of CHD was classified on the basis of the International Statistical Classification of Diseases codes and previous studies ${ }^{(18,19)}$. Exclusion criteria included: (1) inadequate multi-detector computed tomography imaging, due to heavily calcified lesions by visual estimation; (2) a culprit lesion in the left main coronary artery; (3) atrial fibrillation;
(4) malignant disease; (5) dialysis; (6) diabetes mellitus; (7) renal insufficiency.

\section{Blood sample preparation}

After an overnight fasting of the participants, blood $(5 \mathrm{ml})$ was drawn from the antecubital vein into vacutainer tubes containing ethylene diamine tetraacetic acid. Serum was separated from erythrocytes by centrifugation at $2000 \boldsymbol{g}$ at $4^{\circ} \mathrm{C}$ for $10 \mathrm{~min}$. Erythrocytes were washed three times with ice-cold isotonic saline to remove the buffy coat. Membranes were isolated by a modification of Burton's method ${ }^{(20)}$. Briefly, packed cells were lysed with cold distilled water, centrifuged at $20000 \mathrm{~g}$ at $10^{\circ} \mathrm{C}$ for $20 \mathrm{~min}$, and washed several times to eliminate $\mathrm{Hb}$ residues. Whole-blood, serum and erythrocyte membrane samples from each participant were stored at $-80^{\circ} \mathrm{C}$ under liquid $\mathrm{N}_{2}$ until lipid extraction.

\section{Extraction and analysis of fatty acids}

Lipids were extracted by chloroform-methanol (1:1) and methylated by sodium methoxide as described previously ${ }^{(21)}$. Fatty acid methyl esters were analysed by a gas chromatograph (GC 6890 N; Agilent) equipped with a flame ionisation detector, an autosampler injector (7683-B; Agilent) and a fused silica capillary column (CP-Sil 88, $100 \mathrm{~m} \times 0.25 \mathrm{~mm}$ inner diameter, $0 \cdot 20 \mu \mathrm{m}$ film thickness; Varian). The temperature was held at $45^{\circ} \mathrm{C}$ for $4 \mathrm{~min}$, and then ramped to $175^{\circ} \mathrm{C}$ at a flow rate of $13^{\circ} \mathrm{C} / \mathrm{min}$, held for $27 \mathrm{~min}$, and, finally, increased to $215^{\circ} \mathrm{C}$ at a flow rate of $4^{\circ} \mathrm{C} / \mathrm{min}$, held for $35 \mathrm{~min}$.

The levels of five TFA (trans-16:1n-7, trans-16:1n-9, trans$18: 1 n-7$, trans-18: $1 n-9$ and trans-18:2n-6n-9) were calculated from the GC results using normalisation and internal standard methods, as described previously ${ }^{(22)}$. The results were compared with standard fatty acid methyl esters (GLC-463; Nu-Chek Prep, Inc.), with 21:0 fatty acid methyl esters added. Fatty acid profiles were expressed as the percentage of TFA. The TFA index was defined by the following equation:

$$
\begin{aligned}
& \text { TFAindex } \\
& =\exp \left[\sum \frac{\text { trans-16:1n-9+trans- } 18: 1 n-9+\text { trans }-18: 2 n-6 n-9}{\text { trans }-16: 1 n-7+\text { trans }-18: 1 n-7}\right] .
\end{aligned}
$$

In this equation, the numerator adds the levels of industrial TFA (trans-16:1n-9, trans-18:1n-9 and trans-18:2n-6n-9) together, and the denominator adds the levels of ruminant TFA (trans-16:1n-7 and trans-18:1n-7) together. An exponential function was used to make the index stronger and more obvious as the TFA index.

\section{Characteristic data}

Serum lipids (TAG, total cholesterol and HDL), apoA (mainly included apoAI), apoB (included both apoB100 and apoB48) and lipoprotein $\alpha$ were measured in the hospital clinical laboratory with an automatic biochemistry analyser (Beckman 
Table 1. Characteristics of study participants ( $n$ 2713)

(Mean values and standard deviations; number of patients and percentages)

\begin{tabular}{|c|c|c|c|c|c|c|c|c|c|c|c|c|c|c|c|c|c|}
\hline & \multicolumn{4}{|c|}{ ACS $(n 581)$} & \multicolumn{4}{|c|}{ CCAD $(n 631)$} & \multicolumn{4}{|c|}{$\mathrm{HRP}(n 659)$} & \multicolumn{4}{|c|}{$\mathrm{HV}(n 842)$} & \multirow[b]{2}{*}{$P$} \\
\hline & Mean & SD & $n$ & $\%$ & Mean & SD & $n$ & $\%$ & Mean & SD & $n$ & $\%$ & Mean & SD & $n$ & $\%$ & \\
\hline Age (years) & $56 \cdot 78^{\mathrm{C}}$ & $19 \cdot 78$ & & & $48 \cdot 21^{a, b}$ & 8.23 & & & $52.99^{b}$ & $12 \cdot 11$ & & & $46 \cdot 93^{\mathrm{a}}$ & $9 \cdot 34$ & & & $<0.05$ \\
\hline Male & & & 371 & $63 \cdot 86^{a}$ & & & 412 & $65 \cdot 29^{b}$ & & & 420 & $63.73^{b}$ & & & 509 & $60 \cdot 45^{\mathrm{c}}$ & $<0.05$ \\
\hline Tobacco use & & & & & & & & & & & & & & & & & \\
\hline Current (\%) & & & & $20^{\mathrm{a}}$ & & & & $23^{a}$ & & & & $40^{\mathrm{b}}$ & & & & $20^{\mathrm{a}}$ & $<0.001$ \\
\hline Ex $(\%)$ & & & & $69^{c}$ & & & & $31^{a, b}$ & & & & $37^{b}$ & & & & $28^{\mathrm{a}}$ & $<0.001$ \\
\hline $\mathrm{BMI}\left(\mathrm{kg} / \mathrm{m}^{2}\right)$ & $26 \cdot 83^{\mathrm{C}}$ & $2 \cdot 11$ & & & $25 \cdot 30^{\mathrm{b}, \mathrm{c}}$ & 1.98 & & & $23 \cdot 01^{b}$ & 0.95 & & & $21 \cdot 81^{\mathrm{a}}$ & 1.09 & & & $<0.05$ \\
\hline Weight (kg) & $80 \cdot 30$ & 2.98 & & & 75.08 & 3.31 & & & 68.87 & $4 \cdot 20$ & & & 69.79 & 9.18 & & & 0.084 \\
\hline \multicolumn{18}{|c|}{ Waist circumference (cm) } \\
\hline Male & $90 \cdot 12$ & 0.92 & & & $89 \cdot 21$ & 0.32 & & & 80.23 & 1.21 & & & 78.60 & 0.19 & & & 0.201 \\
\hline Female & $82 \cdot 87$ & 1.23 & & & $76 \cdot 30$ & 0.98 & & & $75 \cdot 90$ & 1.63 & & & 72.59 & 1.87 & & & 0.098 \\
\hline College education & & & 120 & $20 \cdot 65$ & & & 210 & 33.28 & & & 298 & $45 \cdot 22$ & & & 321 & $38 \cdot 12$ & 0.121 \\
\hline Blood lipids* & & & & & & & & & & & & & & & & & \\
\hline TAG $(\mathrm{mmol} / \mathrm{l})$ & $2 \cdot 19^{\mathrm{b}}$ & 0.92 & & & $1.85^{\mathrm{b}}$ & 1.03 & & & $1 \cdot 82^{\mathrm{b}}$ & 0.91 & & & $1 \cdot 12^{\mathrm{a}}$ & 1.09 & & & $<0.05$ \\
\hline $\mathrm{TC}(\mathrm{mmol} / \mathrm{l})$ & $6 \cdot 61^{\mathrm{C}}$ & 1.57 & & & $5 \cdot 45^{\mathrm{b}}$ & $2 \cdot 31$ & & & $6 \cdot 14^{\mathrm{C}}$ & 1.22 & & & $4 \cdot 24^{\mathrm{a}}$ & 1.22 & & & $<0.05$ \\
\hline HDL (mmol/l) & 0.90 & 0.19 & & & 1.01 & 1.01 & & & $1 \cdot 19$ & 0.91 & & & 1.08 & 0.53 & & & 0.059 \\
\hline LDL (mmol/l/) & $3.58^{\mathrm{b}}$ & $2 \cdot 11$ & & & $3.51^{\mathrm{b}}$ & 1.33 & & & $3 \cdot 62^{b}$ & 0.89 & & & $2 \cdot 35^{\mathrm{a}}$ & 0.86 & & & $<0.001$ \\
\hline ApoA $(g / l)$ & 1.87 & 0.58 & & & 1.76 & 0.19 & & & 1.29 & 0.21 & & & 1.30 & 0.28 & & & 0.098 \\
\hline ApoB (g/l) & 1.26 & 0.02 & & & 1.01 & 0.71 & & & 1.29 & 0.23 & & & 0.99 & 0.07 & & & 0.061 \\
\hline $\mathrm{Lp}-\alpha(\mathrm{mg} / \mathrm{l})$ & 178.89 & 9.81 & & & $150 \cdot 18$ & $8 \cdot 12$ & & & 134.19 & 7.31 & & & $140 \cdot 31$ & 31.23 & & & 1.981 \\
\hline \multicolumn{18}{|c|}{ Blood pressure $(\mathrm{mmHg})$} \\
\hline Systolic & $129 \cdot 21$ & $3 \cdot 21$ & & & $139 \cdot 12$ & $4 \cdot 22$ & & & $131 \cdot 11$ & $2 \cdot 11$ & & & 113.98 & 1.01 & & & 0.061 \\
\hline Diastolic & 86.31 & 5.31 & & & $82 \cdot 21$ & 1.09 & & & 80.98 & 1.19 & & & 81.56 & $2 \cdot 11$ & & & 0.059 \\
\hline Glucose (mmol/l) & $6 \cdot 21$ & 0.31 & & & 5.91 & 0.11 & & & $6 \cdot 10$ & 0.92 & & & $5 \cdot 27$ & 0.78 & & & 0.073 \\
\hline
\end{tabular}

ACS, acute coronary syndrome; CCAD, chronic coronary artery disease; HRP, high-risk population; HV, healthy volunteers; TC, total cholesterol; Lp- $\alpha$, lipoprotein $\alpha$.

*Blood lipids tested include: TAG; TC; HDL-cholesterol (HDL); LDL-cholesterol (LDL); apoA (mainly including apoAl); apoB (including both apoB100 and apoB48); Lp- $\alpha$. 
CX9). Levels of LDL were calculated by the Friedewald equation. Other standard risk factors, including age, sex, BMI, tobacco use, duration of diabetes mellitus, systolic/diastolic blood pressure, waist circumference and history of diagnosed hypertension, were collected as variables (Table 1).

\section{Statistical analysis}

The 10-year RP of CHD was calculated on the basis of the Cox proportional hazards multivariate model formulation and the Framingham risk equation $^{(23)}$. The risk score was defined as

$$
\begin{aligned}
f_{\text {(group) }}(X, M)= & \beta_{1}\left(X_{1}-M_{1}\right)+\beta_{2}\left(X_{2}-M_{2}\right) \\
& +\beta_{3}\left(X_{3}-M_{3}\right)+\cdots+\beta_{i}\left(X_{i}-M_{i}\right) .
\end{aligned}
$$

Furthermore, the 10-year CHD RP was calculated from the following equation:

$$
\mathrm{RP}_{\text {(group) }}=1-S_{0}(t)^{\exp \left(f_{\text {(group) }}[X, M]\right)},
$$

where $\mathrm{RP}_{\text {(group) }}$ is the $\mathrm{CHD}$ probability within the next 10 years of each group (ACS, CCAD, HRP and HV); $S_{0}(t)$ is the hazard function at time $t ; X_{1}, X_{2}, X_{3}, \ldots, X_{i}$ are independent risk factor variables for each individual; $M_{1}, M_{2}, M_{3}, \ldots, M_{i}$ are the average levels of risk factors in each group; $\beta_{1}, \beta_{2}, \beta_{3}, \ldots, \beta_{i}$ are the partial regression coefficients of different risk factors

The estimated partial regression coefficients, hazard ratio and their corresponding 95\% CI are shown in Table 2; the descriptive characteristics are shown in Table 1 . These parameters and data were substituted into equation 2 and the risk score for the ACS group was calculated as $f_{(\mathrm{ACS})}(X, M)$, which, in turn, was substituted into equation 3 and the average 10 -year $\mathrm{RP}_{(\mathrm{ACS})}$ was calculated as $1-0.9876^{\exp \left(f_{(\mathrm{ACS})}[X, M]\right)}$. In the same way, the average 10-year $\mathrm{RP}_{\mathrm{CCAD}}$ of the CCAD group was calculated as $1-0.9821^{\exp \left(f_{(\mathrm{CCAD})}[X, M]\right)}$, the average 10-year $\mathrm{RP}_{(\mathrm{HRP})}$ of the HRP group as $1-0.9781^{\exp \left(f_{(\mathrm{HRP})}[X, M]\right)}$, the average 10 -year $\mathrm{RP}_{(\mathrm{HV})}$ of the $\mathrm{HV}$ group as $1-0.9753^{\exp \left(f_{(\mathrm{HV})}[X, M]\right)}$ (For the equation calculation process, see the Supplementary material, available online).

The assumption of proportional hazards and the calibration were adjusted for confounding factors such as baseline age, sex, smoking and LDL, and verified by the Hosmer and Lemeshow test ${ }^{(24,25)}$. The proportional hazards assumption was considered to be valid when the difference in the $P$ value was $<0.05$.

SPSS for Windows version 18.0 was used to calculate the regression equations and correlation coefficients between the TFA index and the 10-year CHD-RP for the different groups. Bonferroni correction was performed for ANOVA and correlation analysis to verify statistically significant differences with a $P$ value $<0 \cdot 05$.

\section{Results}

\section{Trans-fatty acid profile and index}

The erythrocyte membrane, serum and whole-blood TFA profiles are listed in Table 3. Only in erythrocyte membranes were

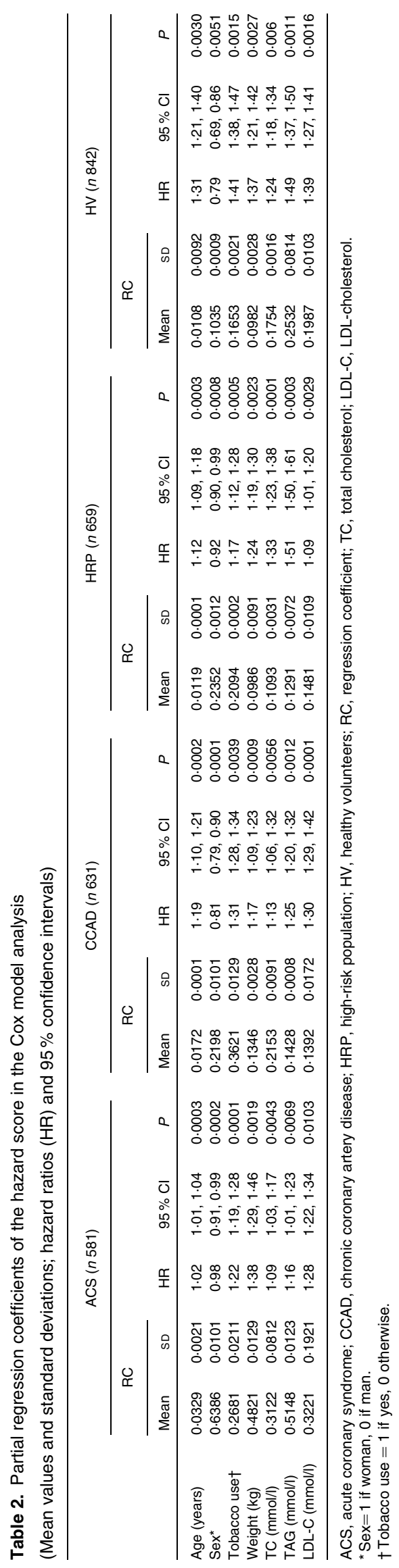


there significant differences in the levels of all TFA isomers, as well as the TFA index among the different groups. The levels of industry-generated isomers (trans-16:1n-9, trans-18: $1 n-9$ and trans-18:2n-6n-9) were the highest in the ACS group, whereas the levels of ruminant-originated isomers (trans-16:1n-7 and trans-18: $1 n-7)$ were the highest in the HV group, and the TFA index showed a significant progressive decrease from the ACS to the HV group. In serum, only the levels of trans- $18: 2 n-6 n-9$ were significantly different between these groups; although the TFA index was high in the ACS group, there was still no significant difference. In whole blood, the levels of both trans-18: $1 n-9$ and trans-18:2n-6n-9 were significantly higher in the ACS group than those in the other groups, but no difference existed in the other groups. In addition, the total industrial and ruminant TFA levels showed no significant differences between the groups.

In the TFA index equation, the total industrial TFA levels in the numerator exhibited an increasing trend from the HV to the ACS group, while the total ruminant TFA levels in the denominator showed a decreasing trend. To unify the incompatible trend of decreased ruminant TFA and increased industrial TFA from the HV to the ACS group, we divided the total industrial TFA levels by the total ruminant TFA levels. This index value represents a coincidental trend of TFA change. In all the four groups, only the erythrocyte membrane TFA index manifested a significant difference. The TFA index in the erythrocyte membrane fraction progressively decreased from $7 \cdot 12$ (SD 1.23) to 5.06 (SD 1.03), 3.11 (SD 0.89) and 1.92 (SD 0.66) in the ACS, CCAD, HRP and HV groups, respectively $(P<0 \cdot 001)$.

\section{CHD risk probability}

The descriptive characteristics of traditional CHD risk factors in each group are described in Table 1. Sex, age, tobacco use and BMI were significantly different between the groups, while waist circumference, weight and education status were not. As for the blood lipids, only total cholesterol, TAG and LDL showed significant differences between the groups. The estimated partial regression coefficients, hazard ratio and their corresponding 95\% CI are shown in Table 2. The risk scores in the four groups were determined as follows: ACS, $f_{(\mathrm{ACS})}(X, M)=31 \%$; CCAD, $f_{(\mathrm{CCAD})}(X, M)=21 \%$; HRP, $f_{(\mathrm{HRP})}(X, M)=13 \% ; \mathrm{HV}, f_{(\mathrm{HV})}(X, M)=4 \%$.

\section{Correlation between trans-fatty acid index and 10-year risk probability}

The comparison between the erythrocyte membrane TFA index and the 10-year CHD RP showed a strong linear correlation $\left(R^{2} 0.9981, P<0.001 ;\right.$ Fig. 1$)$. In contrast, the comparison between the serum or whole-blood TFA index and the 10-year CHD RP showed no significant correlation ( $P>0.05$ and $P>0.01$, respectively; Figs. 2 and 3 ). In each group (HV, HRP, CCAD and ACS), the average erythrocyte membrane TFA index and the 10-year CHD RP coincided with the regression line. 


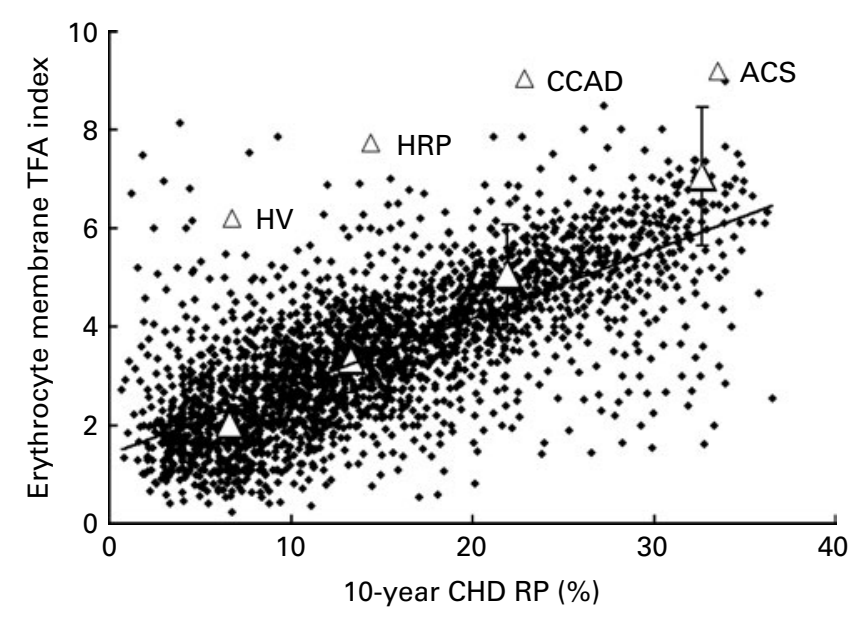

Fig. 1. Linear correlation between the erythrocyte membrane trans fatty acid (TFA) index and the 10-year CHD risk probability (RP) $\left(y=17 \cdot 122 x+1.1365\right.$ and $\left.R^{2} 0.9981\right)$. Values $(\Delta)$ are average TFA index and RP in the healthy volunteers (HV, $n$ 842), high-risk population (HRP $n$ 659), chronic coronary artery disease (CCAD, $n$ 631) and acute coronary syndrome (ACS, $n$ 581) groups, respectively, with standard deviations represented by vertical bars.

\section{Discussion}

\section{Erythrocyte membrane trans-fatty acids reflect the change} in body trans-fatty acids in CHD

The levels of all of the erythrocyte membrane TFA isomers differed significantly between the groups. In contrast, only trans-18:1n-9 and trans-18:2n-6n-9 differed significantly between the whole-blood and serum samples. The probable reasons for this difference were as follows: on the one hand, the serum fatty acid level reflected fat intake over the past few days due to the immediate penetration of dietary fat into the serum ${ }^{(26)}$, and it is hard to get much change in fatty acid profile during a few days, especially TFA; on the other hand, the fatty acid level of erythrocyte membrane reflected chronic lipid storage, as well as the average level of body lipids over the previous 4-12 weeks ${ }^{(27)}$, and there was no fatty acid synthesis, chain elongation or desaturation in the membrane ${ }^{(28)}$. Therefore, erythrocyte membrane TFA reflected the change in body TFA levels in $\mathrm{CHD}^{(8)}$.

\section{Trans-fatty acid index reflects trans-fatty acid hazard on CHD}

The five TFA isomers in the erythrocyte membrane included two n-9 TFA (trans-16:1n-9 and trans-18:1n-9), two $n-7$ TFA (trans-16:1n-7 and trans-18:1n-7) and one doublebonded TFA (trans-18:2n-6n-9). The two n-9 TFA and trans-18:2n-6n-9 primarily originate from partially hydrogenated vegetable oils ('industrial' TFA) ${ }^{(29)}$. The two $n-7$ TFA are generally ruminant-sourced ${ }^{(29)}$, although trans-18:1n-7 may also come from an industrial source. The levels of $n-9$ TFA and trans-18:2n-6n-9 were significantly higher in ACS, which is consistent with the proven detrimental impact of industrial TFA in the promotion and induction of $\mathrm{CHD}$ events from the Nurses' Health Study in 32826 participants and 6 years of follow-up ${ }^{(9)}$. Another study investigating TFA and sudden cardiac death among 86762 women has shown that the levels of trans-18:2 and trans-16:1n-9 were positively associated with myocardial infarction $(P<0 \cdot 001)$ after adjusting for established risk factors and other confounders, and that the trans-18:2 isomer may play a greater role in sudden cardiac death among individuals with clinically manifest atherosclerosis ${ }^{(30)}$.

Remarkably, in contrast, $n-7$ TFA levels were high in the HV group while low in the ACS group, which may result from the ruminant-sourced generation. Ruminant trans-16:1n-7 was associated with trans-18:1n-7 and may be converted into trans-18: $1 n-7$ by the carbochain increase ${ }^{(31)}$. As a major TFA of ruminant fat, trans-18:1n-7 is produced in the rumen and converted in tissues to 9-cis, 11-trans-18:2n-6 CLA by $\Delta 9$-desaturase with an average conversion rate of $19 \%{ }^{(32)}$. 9-cis, 11-trans-18: $2 n-6$ CLA could positively modulate HDL-cholesterol metabolism and enhance reverse cholesterol transport, and prevent the progression of atherosclerosis in humans ${ }^{(33)}$. Hence, the effect of ruminant TFA on CHD may be neutral or somewhat favourable due to the indirect benefit of 9-cis, 11-trans-18:2n-6 CLA ${ }^{(34)}$. However, the industrial TFA are harmful. It has been reported that the consumption of industrial trans-18: $1 n-9$ by LDL receptor-deficient (LDL -/-) mice stimulated atherosclerotic development ${ }^{(35)}$, while consumption of a diet enriched in trans-18:1n-7 reduced cholesterol-induced hyperlipidaemia and atherosclerosis and thus protected against atherosclerotic lesions ${ }^{(36)}$. Chronic trans-18:1n-7 supplementation also significantly abated dyslipidaemia in both the food-deprived and postprandial states in JCR:LA-cp rats due to reductions in intestinal chylomicrons and hepatic de novo lipogenesis pathways ${ }^{(37)}$. Another study investigating the effects of ruminant-derived TFA on immune function in a model of the metabolic syndrome (JCR:LA-cp rats) has shown that vaccenic acid might protect from CVD due to an anti-inflammatory action ${ }^{(38)}$.

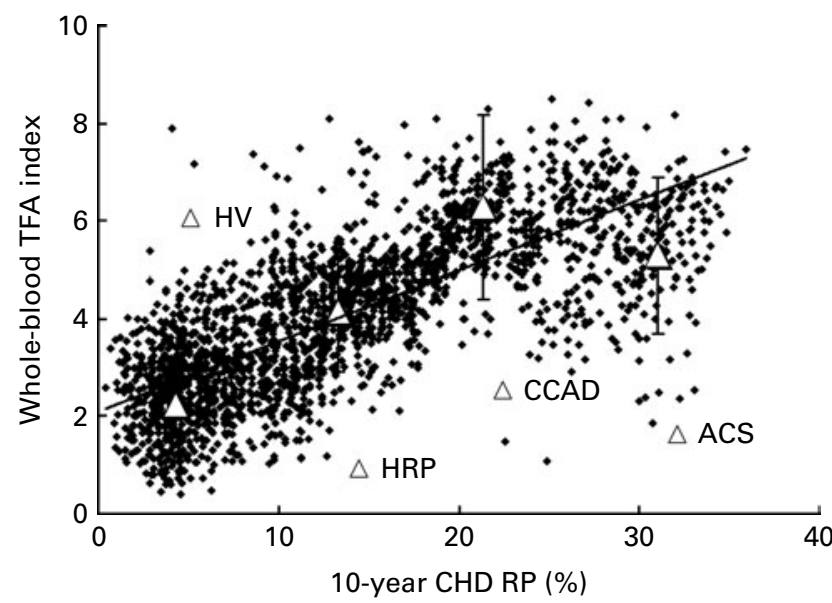

Fig. 2. Linear correlation between the whole-blood trans-fatty acid (TFA) index and the 10-year CHD risk probability (RP) $(y=14.365 x+21.141$ and $R^{2}$ 0.5607). Values $(\Delta)$ are average TFA index and RP in the healthy volunteers (HV, $n$ 842), high-risk population (HRP, $n$ 659), chronic coronary artery disease (CCAD, $n$ 631) and acute coronary syndrome (ACS, $n$ 581) groups, respectively, with standard deviations represented by vertical bars. 


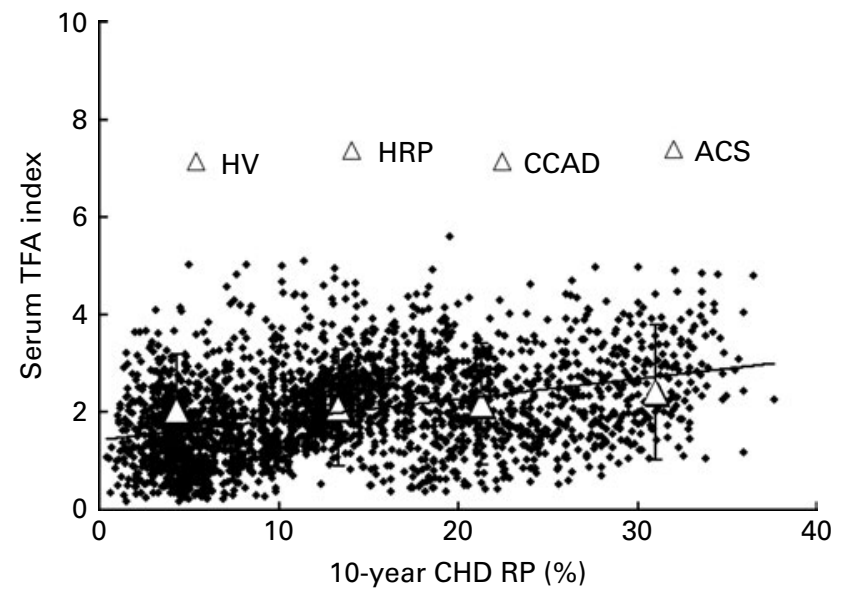

Fig. 3. Linear correlation between the serum trans-fatty acid (TFA) index and the 10-year CHD risk probability (RP) $\left(y=0.104 x+21.81\right.$ and $\left.R^{2} 0.012\right)$. Values $(\Delta)$ are average TFA index and RP in the healthy volunteers (HV $n$ 842), high-risk population (HRP, $n$ 659), chronic coronary artery disease (CCAD, $n$ 631) and acute coronary syndrome (ACS, $n$ 581) groups, respectively, with standard deviations represented by vertical bars.

Although ruminant TFA have been shown to elicit neutral or less detrimental effects, further studies in human subjects are still needed to prove the protective effects

To unify the inverse trend of decreased ruminant TFA and increased industrial TFA from the HV to the ACS group, we divided the total industrial TFA levels by the total ruminant TFA levels. This index value represents a coincidental trend of TFA change. An exponential function was used to make the index stronger and more obvious as the TFA index. The TFA index could estimate the true objective change in TFA levels comprehensively by unifying the disparate effects of different TFA on the body; accordingly, the hazardous level of all TFA isomers on CHD could be evaluated. In addition, it was interesting to note that none of the five TFA isomers showed any significant differences except the TFA index $(P<0.001)$ between the ACS and CCAD groups, indicating that the TFA index could discriminate the acute CHD symptoms of the ACS from the chronic CHD symptoms of CCAD.

Trans-fatty acid index is associated positively with 10-year CHD risk probability

The average TFA index in erythrocyte membranes differed between the groups and increased progressively from the HV to the ACS group. The 10-year CHD RP were 4, 13, 21 and $31 \%$ in the HV, HRP, CCAD and ACS groups, respectively. These values generally reflect the potential probability and pathological severity of CHD. A strong linear correlation was seen between the average TFA index and the 10-year CHD RP ( $R^{2}$ 0.9981; Fig. 1), which suggests that as the erythrocyte membrane TFA level goes up, CHD may get worse.

CHD incidence could be influenced directly or indirectly by TFA through TAG accumulation, vasodilation, inflammation, PG translation and/or platelet aggregation ${ }^{(8,39)}$. Trans-fatty acids elicit an unfavourable effect on the lipoprotein profile by stimulating cholesteryl ester transfer protein activity
( $r$ 0.58, $P<0.005$ ), increasing the LDL level and decreasing the HDL level $(r-0.57, P<0.01)$. These changes may contribute to a more atherogenic lipoprotein profile ${ }^{(40-42)}$. A high intake of TFA could adversely affect endothelial function this would partially explain why the positive relationship between trans-fats and cardiovascular health took precedence over the adverse effects of trans-fats on lipids and lipoproteins $^{(43,44)}$. In addition, a recent study has suggested that trans-18:1n-9 maintains the levels of vascular cell adhesion molecule-I and intercellular cell adhesion molecule-I upregulated by TNF- $\alpha$ or lipase. This kept the human brain microvascular endothelial function at the stimulated phenotype, which could promote $\mathrm{CHD}^{(45)}$.

In summary, the erythrocyte membrane TFA index was proposed as a method to unify the content changes in different TFA isomers and their effects on CHD in one equation. The TFA index manifested a strong and positive linear correlation with the 10-year CHD RP. Although the present study might be limited to the variety of TFA isomers analysed, the results should contribute to further studies on the relationship between TFA and CHD.

\section{Supplementary material}

To view supplementary material for this article, please visit http://dx.doi.org/10.1017/S0007114513000196

\section{Acknowledgements}

The present study was supported by the National Natural Science Foundation of China (30972482), the Academic Leader Program of Jiangxi Province (2008DD00900), the Postgraduate Innovation Funds of Jiangxi Province (YC09A029), the PhD Subject Fund from the Department of Education (20113601120004) and the Natural Science Fund from the Department of Science and Technology of Jiangxi Province (20114BAB214016), China. We deeply appreciate the participation of our colleagues and co-workers at the Department of Cardiology, the First Affiliated Hospital, Department of Cardiology, Medical College, the Second Affiliated Hospital, Nanchang University, and especially the contribution of the research participants to the study. Z.-Y. D. and X.-R. L. initiated, designed, coordinated and, among others, conducted the initial draft board study. J.-N. H., Y.-W. F., R. L. and J. L. carried out the analysis of TFA. J.-T. P., H. S., Q. P. and W.-F. L. screened the patients and collected the clinical data. X.-R. L drafted and completed the manuscript, which was further edited by all the co-authors. All the authors declare that they have no conflicts of interest in relation to the present study.

\section{References}

1. Shearer GC, Pottala JV, Spertus JA, et al. (2009) Red blood cell fatty acid patterns and acute coronary syndrome. PLOS One 4, e5444.

2. Hunter JE, Zhang J \& Kris-Etherton PM (2010) Cardiovascular disease risk of dietary stearic acid compared with trans, 
other saturated, and unsaturated fatty acids: a systematic review. Am J Clin Nutr 91, 46-63.

3. Moyers B, Farzaneh-Far R, Harris WS, et al. (2011) Relation of whole blood $n-3$ fatty acid levels to exercise parameters in patients with stable coronary artery disease (from the heart and soul study). Am J Cardiol 107, 1149-1154.

4. Chavarro JE, Stampfer MJ, Campos H, et al. (2008) A prospective study of trans-fatty acid levels in blood and risk of prostate cancer. Cancer Epidemiol Biomarkers Prev 17, 95-101.

5. Chajes V, Thiebaut ACM, Rotival M, et al. (2008) Association between serum trans-monounsaturated fatty acids and breast cancer risk in the E3N-EPIC study. Am J Epidemiol 167, 1312-1320.

6. Benatar JR, Gladding P, White HD, et al. (2011) Trans-fatty acids in New Zealand patients with coronary artery disease. Eur J Cardiovasc Prev Rehabil 18, 615-620.

7. Lemaitre RN, King IB, Sotoodehnia N, et al. (2010) Endogenous red blood cell membrane fatty acids and sudden cardiac arrest. Metabolism 59, 1029-1034.

8. Oomen CM, Ocke MC, Feskens EJM, et al. (2001) Association between trans fatty acid intake and 10-year risk of coronary heart disease in the Zutphen Elderly Study: a prospective population-based study. Lancet 357, 746-751.

9. Sun Q, Ma J, Campos H, et al. (2007) A prospective study of trans fatty acids in erythrocytes and risk of coronary heart disease. Circulation 115, 1858-1865.

10. Smith BK, Robinson LE, Nam R, et al. (2009) Trans-fatty acids and cancer: a mini-review. Br J Nutr 102, 1254-1266.

11. Bendsen NT, Stender S, Szecsi PB, et al. (2011) Effect of industrially produced trans fat on markers of systemic inflammation: evidence from a randomized trial in women. J Lipid Res 52, 1821-1828.

12. Mozaffarian D (2008) Commentary: ruminant trans fatty acids and coronary heart disease - cause for concern. Int $J$ Epidemiol 37, 182-184.

13. Rungapamestry V, McMonagle J, Reynolds C, et al. (2012) Inter-organ proteomic analysis reveals insights into the molecular mechanisms underlying the anti-diabetic effects of cis-9, trans-11-conjugated linoleic acid in ob/ob mice. Proteomics 12, 461-476.

14. Cassar A, Holmes DR, Rihal CS, et al. (2009) Chronic coronary artery disease: diagnosis and management. Mayo Clin Proc 84, 1130-1146.

15. Alpert JS, Antman E, Apple F, et al. (2000) Myocardial infarction redefined - a consensus document of the Joint European Society of Cardiology/American College of Cardiology Committee for the redefinition of myocardial infarction. J Am Coll Cardiol 36, 959-969.

16. Khot UN, Jia G, Moliterno DJ, et al. (2003) Prognostic importance of physical examination for heart failure in non-ST-elevation acute coronary syndromes: the enduring value of Killip classification. JAMA 290, 2174-2181.

17. Gibbons RJ, Abrams J, Chatterjee K, et al. (2003) ACC/AHA 2002 guideline update for the management of patients with chronic stable angina - summary article - a report of the American College of Cardiology/American Heart Association Task Force on Practice Guidelines (Committee on the Management of Patients with Chronic Stable Angina). Circulation 107, 149-158.

18. O'Rourke RA, Brundage BH, Froelicher VF, et al. (2000) American College of Cardiology/American Heart Association Expert Consensus Document on electron-beam computed tomography for the diagnosis and prognosis of coronary artery disease. J Am Coll Cardiol 36, 326-340.
19. Niu S, Zhao D, Zhu J, et al. (2009) The association between socioeconomic status of high-risk patients with coronary heart disease and the treatment rates of evidence-based medicine for coronary heart disease secondary prevention in China: results from the Bridging the Gap on CHD Secondary Prevention in China (BRIG) Project. Am Heart $J$ 157, 709.e1-715.e1.

20. Burton GW, Ingold KU \& Thompson KE (1981) An improved procedure for the isolation of ghost membranes from human red-blood-cells. Lipids 16, 946-946.

21. Cruz-Hernandez C, Deng ZY, Zhou JQ, et al. (2004) Methods for analysis of conjugated linoleic acids and trans-18: 1 isomers in dairy fats by using a combination of gas chromatography, silver-ion thin-layer chromatography/ gas chromatography, and silver-ion liquid chromatography. J AOAC Int 87, 545-562.

22. Zghibeh CM, Gopal VR, Poff CD, et al. (2004) Determination of trans-arachidonic acid isomers in human blood plasma. Anal Biochem 332, 137-144.

23. Kinlay S, Oconnell D, Evans D, et al. (1992) The validity of estimating heart-disease reduction from a Framingham logistic equation. J Clin Epidemiol 45, 553-560.

24. Lin DY, Wei LJ \& Ying Z (1993) Checking the Cox model with cumulative sums of Martingale-based residuals. Biometrika 80, $557-572$.

25. Harrell FE, Lee KL \& Mark DB (1996) Multivariable prognostic models: issues in developing models, evaluating assumptions and adequacy, and measuring and reducing errors. Stat Med 15, 361-387.

26. Sepulveda JL, Tanhehco YC, Frey M, et al. (2010) Variation in human erythrocyte membrane unsaturated fatty acids correlation with cardiovascular disease. Arch Pathol Lab Med 134, 73-80.

27. Katan MB, Deslypere JP, vanBirgelen APJM, et al. (1997) Kinetics of the incorporation of dietary fatty acids into serum cholesteryl esters, erythrocyte membranes, and adipose tissue: an 18-month controlled study. J Lipid Res 38, 2012-2022.

28. Pittman JG \& Martin DB (1966) Fatty acid biosynthesis in human erythrocytes: evidence in mature erythrocytes for an incomplete long chain fatty acid synthesizing system. $J$ Clin Invest 45, 165-172.

29. Micha R, King IB, Lemaitre RN, et al. (2010) Food sources of individual plasma phospholipid trans fatty acid isomers: the Cardiovascular Health Study. Am J Clin Nutr 91, 883-893.

30. Chiuve SE, Rimm EB, Manson JE, et al. (2009) Intake of total trans, trans-18:1, and trans-18:2 fatty acids and risk of sudden cardiac death in women. Am Heart J 158, 761-767.

31. Or-Rashid MM, Wright TC \& McBride BW (2009) Microbial fatty acid conversion within the rumen and the subsequent utilization of these fatty acids to improve the healthfulness of ruminant food products. Appl Microbiol Biotechnol 84, 1033-1043.

32. Turpeinen AM, Mutanen M, Aro A, et al. (2002) Bioconversion of vaccenic acid to conjugated linoleic acid in humans. Am J Clin Nutr 76, 504-510.

33. Komori H, Arai H, Kashima T, et al. (2008) Coexpression of CLA-1 and human PDZK1 in murine liver modulates HDL cholesterol metabolism. Arterioscler Thromb Vasc Biol 28, 1298-1303.

34. Gomez-Cortes P, Tyburczy C, Brenna JT, et al. (2009) Characterization of cis-9 trans-11 trans-15 C18:3 in milk fat by GC and covalent adduct chemical ionization tandem MS. J Lipid Res 50, 2412-2420. 
35. Bassett CMC, McCullough RS, Edel AL, et al. (2009) Transfatty acids in the diet stimulate atherosclerosis. Metabolism 58, 1802-1808.

36. Bassett CMC, Edel AL, Patenaude AF, et al. (2010) Dietary vaccenic acid has antiatherogenic effects in LDLr-/mice. J Nutr 140, 18-24.

37. Wang Y, Jacome-Sosa MM, Ruth MR, et al. (2009) Trans-11 vaccenic acid reduces hepatic lipogenesis and chylomicron secretion in JCR:LA-cp rats. J Nutr 139, 2049-2054.

38. Blewett HJ, Gerdung CA, Ruth MR, et al. (2009) Vaccenic acid favourably alters immune function in obese JCR:LA-cp rats. Br J Nutr 102, 526-536.

39. de Lorgeril M \& Salen P (2004) Use and misuse of dietary fatty acids for the prevention and treatment of coronary heart disease. Reprod Nutr Develop 44, 283-288.

40. Abbey M \& Nestel PJ (1994) Plasma cholesteryl ester transfer protein-activity is increased when trans-elaidic acid is substituted for cis-oleic acid in the diet. Atherosclerosis 106, 99-107.
41. Katan MB, Zock PL \& Mensink RP (1995) Trans-fatty-acids and their effects on lipoproteins in humans. Annu Rev Nutr 15, 473-493.

42. Williams PT (2012) Fifty-three year follow-up of coronary heart disease versus HDL2 and other lipoproteins in Gofman's Livermore Cohort. J Lipid Res 53, 266-272.

43. Harvey KA, Arnold T, Rasool T, et al. (2008) Trans-fatty acids induce pro-inflammatory responses and endothelial cell dysfunction. Br J Nutr 99, 723-731.

44. Bionaz M, Thering BJ \& Loor JJ (2012) Fine metabolic regulation in ruminants via nutrient-gene interactions: saturated long-chain fatty acids increase expression of genes involved in lipid metabolism and immune response partly through PPAR-alpha activation. Br J Nutr 107, 179-191.

45. Sanadgol N, Mostafaie A, Bahrami G, et al. (2010) Elaidic acid sustains LPS and TNF-alpha induced ICAM-1 and VCAM-I expression on human bone marrow endothelial cells (HBMEC). Clin Biochem 43, 968-972. 